\title{
Ground State Entanglement in One Dimensional Translationally Invariant Quantum Systems
}

\author{
Sandy Irani* \\ Computer Science Department \\ University of California, Irvine, USA
}

November 27, 2018

\begin{abstract}
We examine whether it is possible for one-dimensional translationally-invariant Hamiltonians to have ground states with a high degree of entanglement. We present a family of translationally invariant Hamiltonians $\left\{H_{n}\right\}$ for the infinite chain. The spectral gap of $H_{n}$ is $\Omega(1 / \operatorname{poly}(n))$. Moreover, for any state in the ground space of $H_{n}$ and any $m$, there are regions of size $m$ with entanglement entropy $\Omega(\min \{m, n\})$. A similar construction yields translationally-invariant Hamiltonians for finite chains that have unique ground states exhibiting high entanglement. The area law proven by Hastings [Has07] gives a constant upper bound on the entanglement entropy for 1D ground states that is independent of the size of the region but exponentially dependent on $1 / \Delta$, where $\Delta$ is the spectral gap. This paper provides a lower bound, showing a family of Hamiltonians for which the entanglement entropy scales polynomially with $1 / \Delta$. Previously, the best known such bound was logarithmic in $1 / \Delta$.
\end{abstract}

\section{Introduction}

Understanding and quantifying entanglement in quantum systems is a central theme in quantum information science. On one hand quantum entanglement is a valuable resource that enables novel computation and communication. On the other hand, the fact that some quantum systems have bounded entanglement accounts for the success of computational methods such as DMRG in finding ground states and simulating dynamics [Whi92, Whi93, Sch05, Vid03, Vid04]. We examine one dimensional quantum systems and ask what is the minimal set of properties a system must have in order to exhibit a high degree of ground state entanglement. In particular, do symmetries such as translational invariance limit entanglement?

We present two closely related constructions. The first is a single Hamiltonian term which acts on two 21-dimensional particles. When the term is apllied to each neighboring pair of particles in a chain of $n$ particles, the resulting Hamiltonian has a unique ground state and a spectral gap of $1 / \operatorname{pol} y(n)$. We show that the entropy of the ground state when traced down to a linear number of particles on either end of the chain scales linearly with $n$. If we allow ourselves boundary conditions in the form of a different singleparticle term applied to the first and last particles of the chain, the Hamiltonian is frustration-free. The boundary conditions can be removed with an additional term applied to all the particles in the chain. With the additional term, the resulting Hamiltonian is no longer frustration free.

The second construction is a family of translationally invariant Hamiltonians $\left\{H_{n}\right\}$. When applied to any cycle whose size is a multiple of $n$ or an infinite chain of particles, the spectral gap is $1 /$ poly $(n)$. The ground state is no longer unique, but for any state in the ground space and any $m$, a constant fraction of the intervals of length $m$ have entanglement entropy that is $\Omega(\min \{m, n\})$. Moreover, there exists a state in

*E-mail: irani@ics.uci.edu. Partially supported by NSF Grant CCR-0514082 and CCF-0916181. Part of this work was done while the author was visiting the Institute for Quantum Information at Caltech. 
the ground space which is translationally invariant and has the property that every interval of length $m$ has entanglement entropy which is $\Omega(\min \{m, n\})$. Nagaj has shown a way to reduce the number of particles to $14[\overline{\mathrm{Nag}}]$.

This paper builds on recent work examining the computational power of one dimensional quantum systems in which it is shown that it is possible to perform universal adiabatic quantum computation using a 1D quantum system [AGIK09]. In addition, it is shown that the problem of determining the ground state of a one dimensional quantum system with nearest neighbor interactions is QMA-complete. Both results make critical use of position-dependent terms in the Hamiltonian. The intuition that symmetry in quantum systems is both natural and might lead to more tractable systems, has lead researchers to examine whether this construction can be made translationally invariant. For instance, [NW08] gives a 20-state translationinvariant modification of the construction (improving on a 56-state construction by [JWZ07]) that can be used for universal 1-dimensional adiabatic computation. These modifications require that the system be initialized to a particular configuration in which the state of each particle encodes some additional information. The terms of the Hamiltonian, although identical, act differently on different particles depending on their initial state. The ground state is therefore degenerate and one determines which ground state is reached by ensuring that the system starts in a particular state. Liu et. al. [LCV07] show that the $N$-representability problem on fermions is QMA-complete. Since the states occupied by fermionic systems are anti-symmetric, all two-particle reduced density matrices are identical. However, the dimension of the two-particle matrices can grow polynomially with $N$, whereas we consider a constant number of states per particle. Kay [Kay08] gives a construction showing that determining the ground energy of a one dimensional nearest-neighbor Hamiltonian is QMA-complete even with all two-particle terms identical, though the construction requires position-dependent one-particle terms. It is not clear how one would eliminate both the varying terms on individual particles and the degeneracy of the ground state and still obtain a complexity result. In particular, it is not clear how a single Hamiltonian term with bounded precision acting on a pair of particles with constant dimension would encode a circuit or an input instance. However, it is still natural to examine ground state entropy under these limitations.

This work also relates to the area law for one dimensions proven by Hastings [Has07]. An area law asserts that in ground states of local Hamiltonians, the entanglement entropy of the reduced state of a subregion should scale with the boundary of the region as opposed to the volume of the region. In one dimensions, the bounding area of a contiguous region is comprised only of the two endpoints of the segment, so the area law says that the entropy of entanglement should be independent of the size of the region. The area law for one-dimensional systems proven by Hastings depends exponentially on $1 / \Delta$, where $\Delta$ is the spectral gap. Gottesman and Hastings raised the question as to whether this dependence on $\Delta$ is tight and towards this end gave a family of Hamiltonians on the infinite chain whose entanglement entropy scales as $\Omega\left((-\Delta \log \Delta)^{-1 / 4}\right)$. Previously studied systems have the property that the entropy of all intervals is bounded by a constant times $\log (1 / \Delta)$.

The results presented in this paper independently provide a lower bound of this kind, although the two sets of results have different features resulting from the different motivation of the authors. The [GH08] construction is not translationally invariant as this is not required for the area law. However, it is simpler, uses fewer states and the lower bound on entanglement as a function of the spectral gap is a larger polynomial $\left(\Omega\left((-\Delta \log \Delta)^{-1 / 4}\right)\right.$ as opposed to $\Omega\left((\Delta)^{-1 / 12}\right)$ ). Much of the effort in the construction presented here stems from designing a translationally invariant Hamiltonian. In both constructions, the ground state achieves high entanglement on some but not all of the regions. Technically, this is valid for a lower bound on the area law since an area law must give an upper bound on the entanglement entropy for all regions. Nonetheless, Gottesman and Hastings point out that their construction can be augmented, using 81 instead of 9 states, to produce a ground state with high entanglement on every sufficiently large region. Note that high entanglement entropy means polynomial in $1 / \Delta$. The entropy will not grow as the region size tends towards infinity as this would violate Hastings' upper bound. Finally there is the fact that the ground space for the construction presented here is degenerate. Note that this degeneracy is fundamentally different from the 
degeneracy in the constructions of [NW08] and [JWZ07] discussed above in that every state in the ground space exhibits the desired entanglement properties. There does not seem a way to break the degeneracy in this particular construction using local, translationally invariant rules. The basis of the ground space consists of states which are translations of each other along the infinite chain. Since the basis states for the ground space of $H_{n}$ are periodic with period $n$, the dimension of the ground space is $n$. If one is willing to forego translational invariance, we could use the construction for the finite chain presented here to design a Hamiltonian for the infinite chain with a unique ground state by simply repeating copies of the Hamiltonian for a chain of length $n$, side by side. It should be noted that Hastings' upper bound is only proven for Hamiltonians with degenerate ground states. It is not clear whether the proof can be extended when the degeneracy of the ground state is polynomial in $1 / \Delta$, where $\Delta$ is the difference between the lowest and second lowest eigenvalues of the Hamiltonian.

In the constructions presented here and in [GH08], each Hamiltonian depends on the parameter $n$ which in turn gives a fixed upper bound for the entanglement entropy that can be achieved for any region. It is unclear whether there is a way to achieve entanglement entropy that is linear in the region size simultaneously for all region sizes. In the only known examples of 1D ground states for which the entanglement entropy grows asymptotically with the size of the region, the entropy depends logarithmically on the region size [VLRK03, ECP08]. It has been conjectured that for any translationally invariant pure state, the entropy of entanglement of a contiguous set of $n$ particles is sublinear as $n$ grows [FZ05]. This conjecture does not require that the state be a ground state of a Hamiltonian (translationally invariant or otherwise). It is simply a question about what can be achieved by a quantum state. In this sense it should be easier to achieve high entanglement. On the other hand, the conjecture requires that the state itself be translationally invariant.

\subsection{Outline of Techniques}

We begin with an overview of the construction for the finite chain, much of which will also be used for the cycle and infinite chain. We will have 21 states on each site consisting of 2-state subsystems which hold a qubit of data and 1-state subsystems. We use the term configuration to refer to an arrangement of the states on a line without regard to the value of the data stored in the qubit subsystems. The Hamiltonian applied to each pair of particles will consist of a sum of terms of which there are two types. The first type will have the form $|a b\rangle\langle a b|$ where $a$ and $b$ are single particle states. We call these illegal pairs as it has the effect of adding an energy penalty to any state which has a particle in state $a$ to the immediate left of a particle in state $b$. We will say a configuration is legal if it does not contain any illegal pairs. The second type of term will have the form: $\frac{1}{2}(|a b\rangle\langle a b|+| c d\rangle\langle c d|-| a b\rangle\langle c d|-| c d\rangle\langle a b|)$. These terms enforce that for any eigenstate with zero energy, if there is a configuration $A$ with two neighboring particles in states $a$ and $b$, there must be a configuration $B$ with equal amplitude that is the same as $A$ except that $a$ and $b$ are replaced by $c$ and $d$. Even though these terms are symmetric, we associate a direction with them by denoting them as $a b \rightarrow c d$. These terms will be referred to as transition rules. We will say that configuration $A$ transitions into configuration $B$ by rule $a b \rightarrow c d$ if $B$ can be obtained from $A$ by replacing an occurrence of $a b$ with an occurrence of $c d$. We say that the transition rule applies to $A$ in the forward direction and applies to $B$ in the backwards direction. We will choose the terms so that for any legal configuration, at most one transition rule applies to it in the forward direction and at most one rule applies in the backwards direction. Thus, a ground state consists of an equal superposition of legal configurations such that there is exactly one transition rule that carries each configuration to the next. So far what we have described is a standard procedure in QMA-completeness results with the chain of configurations in the ground state corresponding to the execution of the circuit through time [KSV02, KKR06, $\mathrm{AvDK}^{+} 04$, OT08]. For a one dimensional system, we have a small set of designated states called control states and we enforce that any legal configuration has exactly one particle in a control state. The transition rules apply only to the control state and a particle to the immediate left or right, possibly moving the control state left or right by one position, much like the head of a Turing Machine. This idea was also employed in [AGIK09].

In the construction we present here, particles on the left half of the chain start in a 2-state subsystem 
that each encode a qubit in state $|+\rangle$. The control state will act as a courier, first getting entangled with a qubit on the left end, and then moving to the right part of the chain. There it gets swapped with a qubit state, creating an entangled qubit pair which spans the center of the chain. In each round trip made by the control state, the number of entangled pairs increases by one and eventually the number of entangled pairs spanning the center of the chain is roughly half the number of particles in the chain. When the qubit value of a particle on the left has been recorded by (or entangled with) the qubit value stored with the control particle, it transitions to a two-state subsystem which we represent by the symbol $(e$ ). Similarly when the particle on the right becomes entangled with the qubit value of the control state, it transitions to a two-state subsystem represented by the symbol $(E)$. Thus the particles in these states build up over time on the two ends of the chain. The transition rules ensure that the courier changes direction as soon as it hits a particle in state (e) or (E). The process is illustrated below with overbrace spanning the newly created entangled pair. In the actual construction more particle states will be required to ensure that the process proceeds as depicted.

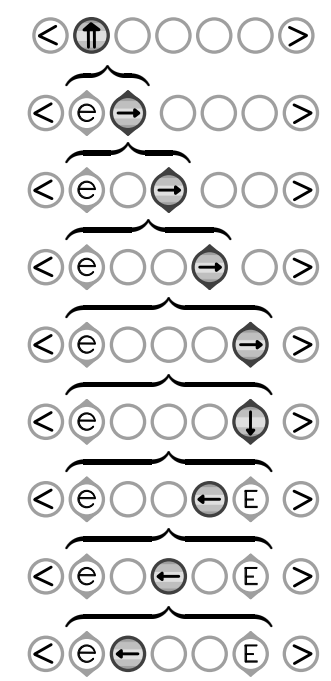

Once the construction is given in detail, it is fairly evident that it results in a high entanglement ground state and the main difficulty is to establish that this ground state is unique. Thus, additional constraints are required to give energy penalties to configurations that deviate from this plan. As was the case with the one dimensional QMA-completeness construction of [AGIK09], we are not able to eliminate every undesirable configuration with local checks and we need to show that some bad configurations are ruled out because they must eventually evolve (via forward or backwards transitions) to a configuration which can be eliminated by local constraints. For the problem addressed here, we need some means of enforcing that entangled pairs actually span the center of the chain instead of spanning some boundary to the far left or the far right as this could severely limit the number of entangled pairs. We also need to enforce the condition that particles initially storing the $|+\rangle$ state to be entangled with other particles further down the chain occur on the left half of the chain. This could be easily managed with different terms on the left half and the right half of the chain. However, since we insist on uniform terms, we enforce these conditions by showing that violating states will evolve to illegal states. For example, if the number of particles in state $(e)$ on the left is less than the number of particles in state $(E)$ on the right, we show that this state will evolve via backwards transitions to a state with a collection of particles in state $(E)$ on the right and no particles in state $(\odot)$ on the left. The first and last particles in the chain will be in special delimiter states (with $<$ at the left end and $>$ at the right end) which will be used to detect this occurrence and trigger an energy penalty. This raises a new problem of how to make sure only the end particles are in these delimiter states. This is done by adding a penalty for any particle that is in a state which is different from one of the delimiter states. Finally, we add even greater penalties for any pair of the form $X \odot$ or $\gg X$ which ensures that only the leftmost particle will be in state (<) and only the rightmost particle will be in state $>$. 
The construction for the finite chain makes use of the fact that the endpoints of the chain have only one neighbor. When we move to the cycle we not longer have these special particles. We change the Hamiltonian for the cycle by allowing the pair $\gg \diamond$ with the effect that the set of legal states become sequences of segments bracketed on either side. The legal states look like the following type of sequence wrapped around the cycle:

$$
<\cdots \gg<\cdots \otimes<\cdots \otimes<\cdots>
$$

Suppose we fix the locations of the $<$ and $\gg$ sites and consider the space of states with those locations fixed. The Hamiltonian $H_{n}$ will be closed on that space which allows us to analyze every such subspace separately. Finally a term is added that gives an energy penalty if there is a sequence from a $\diamond$ site to a $>$ site whose length is not exactly equal to $n$. Thus, the ground state for a cycle of length $t n$ will be $t$ copies of the ground state for a finite chain of length $n$ tensored together. There are $n$ such global states, each a rotation of the others. We will show that the bounds on the spectral gap and the entanglement entropy are independent of $t$, so as $t$ goes towards infinity, the bounds will still hold which means that they also hold for the infinite chain.

\section{The Basic Construction on a Finite Chain}

The 21 states in each site consist of 2-state subsystems (different versions of a qubit holding data), represented by elongated shapes (e.g., $\Theta$ ), and 1-state subspaces, represented by round shapes (e.g., $\Theta$ ). 11 Three of the 2-state subsystems and three of the 1-state subsystems will be control sites, which will be represented by dark shapes and can be thought of as pointers on the line that trigger transitions. Light-colored shapes represent a site that is inactive, waiting for the active site to come nearby. There will only be one control site in any legal configuration. Particles in states denoted by lower case letters will always be to the left of the control site and particles in states denoted by upper case letters will be to the right of the control state. When needed, we will indicate the value of the qubit stored in a 2-state subsystem with a subscript indicating the state, such as $\Theta_{+}$or $\Theta_{1}$. We have the following types of states:

$\underline{\text { Inactive states }}$

(E), (e): Qubits entangled with another site

(U), (U): Qubits unentangled with another site

(W, (w): Particles waiting to be entangled

(<), (>: The left and right end delimiters
Control states

$\Theta, \Theta$ : Right-moving control states

$\Theta$ : Left-moving control state

(1), (11: Left-end turning control states

(1): Right-end turning control state

We start by introducing the set of transition rules. Unless otherwise specified, a rule applied to a 2state subsystem is summed over all possible values for the qubits, with the control particle keeping its value and the non-control particle keeping its value. For example, the rule $\Theta(\cup) \rightarrow(U)$ would be the sum of $\Theta_{x}\left(\cup_{y} \rightarrow \Theta_{y} \Theta_{x}\right.$ over all possible values for $x, y \in\{0,1\}$. The exceptions to this are made explicit in the set of rules below. The sum of all the resulting terms is denoted by $H_{\text {trans }}$.

The rules involving sites with single arrows are used throughout most of the evolution of the configurations. Rules involving sites with double arrows occur only during the first iteration of the construction and are used to check the validity of the starting configuration.

Transition Rules:

1. $\Theta(\mathbb{U}) \rightarrow(U), \Theta \mathbb{W} \rightarrow(\mathbb{W} \Theta$ : Sweeping to the right past $(\mathrm{U})$ and $\mathbb{W}$ sites, transforming them to $(\mathrm{U})$ and $(\omega)$.

${ }^{1}$ This notation, which has been adapted for the construction presented here, was developed in AGIK09 in collaboration with Oded Regev. 
2. $(A) \rightarrow(1)(E)$ : Control turns once an entangled site is reached on the right end.

3. (1) (D) $\rightarrow \Theta \mathbb{E}_{x}$ : Control starts moving left and transfers its qubit state to the $(\mathbb{E})$.

4. $\mathrm{W} \Theta \rightarrow \Theta \mathbb{W},(\mathrm{U}) \Theta \rightarrow \Theta(\mathrm{U})$ : Control sweeps to the left past $(\mathrm{U})$ and $\mathbb{W}$ sites, transforming them to (U) and (W) sites.

5. $\odot \ominus \rightarrow(\ominus$ : Control turns once an entangled site is reached on the left end.

6. (1)(U) $x \rightarrow\left(\Theta \Theta_{x} \Theta_{x}\right.$ : Control starts moving right. Qubit values of $(\ominus)$ and $\Theta$ become entangled.

7. (11)(U) $x \rightarrow \Theta_{x} \Theta_{x}$ : Control starts moving right. Qubit values of $(\ominus$ and $\Theta$ become entangled.

8. $\Theta(\mathbb{U}) \rightarrow(\mathbb{U}) \Theta, \Theta \mathbb{W} \rightarrow \mathbb{W} \Theta$ : Sweeping to the right past (U) and $\mathbb{W}$, transforming them to (U) and (w).

9. $\Theta \gg \rightarrow(1) \gg$ : Control turns once the right end delimeter is reached.

Rules 6 and 7 above are the rules that create the entanglement in the construction. Later in the construction, we will add terms which enforce that for any state in the support of a ground state, if a particle is in state (U), then the value stored in the qubit of that 2 -state subsystem must be $|+\rangle$. The action of transition rules 6 and 7 on a state of this kind create an entangled pair:

$$
\begin{aligned}
& \text { (1) }\left(\frac{1}{\sqrt{2}} \text { (U) }_{0}+\frac{1}{\sqrt{2}}\left(\mathrm{U}_{1}\right) \rightarrow \frac{1}{\sqrt{2}}\left(\Theta_{0} \Theta_{0}+\Theta_{1} \Theta_{1}\right) .\right. \\
& \text { (II) }\left(\frac{1}{\sqrt{2}} \text { (U) }_{0}+\frac{1}{\sqrt{2}}\left(\mathrm{U}_{1}\right) \rightarrow \frac{1}{\sqrt{2}}\left(\Theta_{0} \Theta_{0}+\Theta_{1} \Theta_{1}\right) .\right.
\end{aligned}
$$

We wait to introduce the rule that enforces that a (U) particle must be in the state (U) because it will be convenient for the first part of the proof to work with standard basis states. A state in the standard basis is first specified by its configuration and then by a 0 or 1 value for each 2 -state subsystem in the configuration. Thus, a standard basis state is represented by a string of symbols from the set

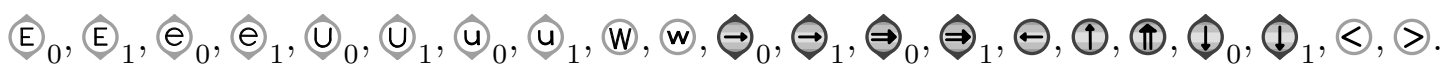

A configuration is represented by a string of symbols from this set without the 0 and 1 subscripts specified. The transition rules and illegal pairs are all specified with respect to standard basis states.

We are now ready to describe the evolution of configurations in the target ground state. We assume that we start with a configuration in the following form $<\mathbb{( 1 1}()^{m} \mathbb{W}^{m} \nabla$, where $n=2 m+3$. (We will eventually prove that the low energy states exist only when $n$ is odd). The construction is illustrated with a small example below to show what happens as each rule is applied:

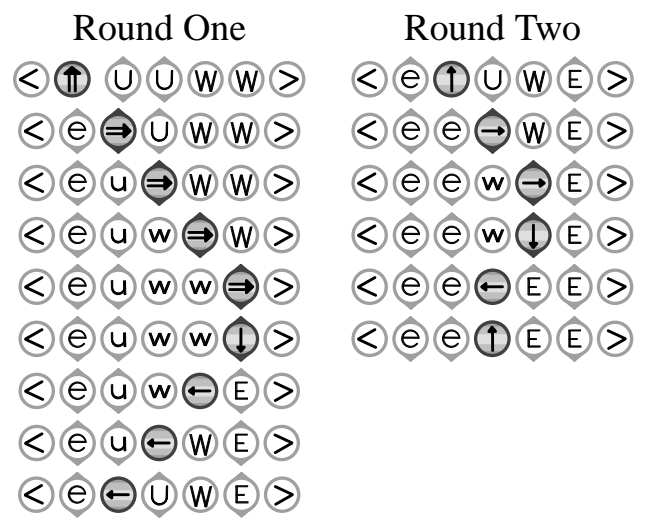


Now we describe a set of terms that are designed to ensure that the state corresponding to the evolution of configurations shown above is the only low energy state. The constraints are expressed in terms of illegal pairs (pairs of states which cause an energy penalty if they appear side by side in a configuration). It is sometimes convenient to describe a set of states informally such as (UPPERCASE) which denotes any state represented by an upper case letter. The label for each set is indicated below along with the set of states in that set.

\begin{tabular}{|c|c|}
\hline (anything) & the set of all states \\
\hline (lowercase) & $\{(\Theta),(\mathrm{U}, \mathbb{(})\}$ \\
\hline (UPPERCASE, $\gg$ ) & $\{(\mathrm{E}),(\mathrm{U}),(\mathrm{W}),(\})$ \\
\hline (lowercase, $(<)$ & $\{(\mathrm{e}, \mathrm{(4)}, \mathrm{\uplus}, \mathrm{e}\}$ \\
\hline (UPPERCASE) & $\{(\mathrm{E}),(\mathrm{U}), \mathrm{W})\}$ \\
\hline (control) & $\{\Theta, \Theta, \Theta,(1), \mathbb{1},(1)\}$ \\
\hline
\end{tabular}

Illegal Pairs:

1. $\gg$ (anything), (anything) $\diamond$ : if $\diamond$ is in the system at all, must be at the right. If $\odot$ is in the system at all, it must be at the left end.

2. (UPPERCASE)(lowercase), (Control) (lowercase), (UPPERCASE) (Control) : Lower case sites before control sites before upper case sites.

3. (Control) (Control) : At most one control site.

4. (lowercase, $\odot)$ ) (UPPERCASE, $\diamond)$ ): Lower case and $\odot$ sites must be buffered from upper case and ( sites by a control site.

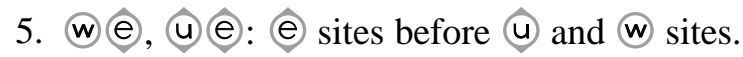

6. (E) (W), (E)(U): (W) and (U) sites before (E) sites.

7. (U) (1), (U) (1), (1) (1), ( (II): Control turns at the left end of unentangled and waiting sites.

8. (1) (U), (1) (W): Control turns at the right end of unentangled and waiting sites.

9. (W) (U), (W) (U): (U) and (U) sites should come before (W) and (W) sites.

10. $\Theta(E), \Theta \gg$ : Will be used to enforce the correct initial configuration.

11. $<(1),(e)(\mathbb{1}, \mathbb{1}(\mathbb{E})$ : Will be used to enforce correct initial configuration.

12. (1) (W), (U) (1), (C) (1): Will ensure that the number of sites in (U) and (U) is same as the number of sites in $(\mathbb{W}$ or $(\mathbb{W}$.

13. $\bigodot_{0} \Theta_{1}, \bigodot_{1} \Theta_{0}$ : Will ensure that pairs of qubits are properly entangled.

14. $\bigodot_{0} \Theta_{1}, \bigodot_{1} \Theta_{0}$ : Will ensure that pairs of qubits are properly entangled.

To define the set of terms arising from the illegal pairs as they act on states (and not just 2-state subsystems), we simply sum over all combinations of qubit values, except for constraints in items 13 and 14 which 
are explicitly specified. For example, the illegal pair (4) (e) gives rise to the term $\mid(4)(e)\langle(\Theta)(e)|$ which is then is expanded as follows:

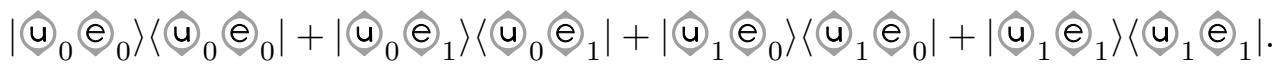

The resulting term obtained from adding all the constraints above is $H_{\text {legal }}$.

Although we will ultimately insist that legal configurations do not contain any illegal pairs, it will be convenient to work with a larger set of configurations/states which only omit illegal pairs listed in items 1 through 8 .

Definition 2.1 A configuration is said to be legal if it has no illegal pairs listed in items 1 through 12 . (The illegal pairs in items 13 and 14 apply only to states). A state is legal if it has no illegal pairs. A configuration or state is well-formed if it does not contain any of the illegal pairs listed in items 1 through 8.

We start by characterizing the set of well-formed configurations. In doing so, we will use the following notation: $\Theta^{*}$ will denote a sequence of sites in state $\Theta$ of arbitrary (possibly zero) length. $(\Theta / \Theta / \Theta)$ is a single state which is either $\Theta$ or $\Theta$ or $\Theta$. $\epsilon$ will denote an empty string of symbols.

Lemma 2.2 The set of well-formed configurations are those configurations which conform to one of the expressions below or any substring of one of the expressions below:

$$
\begin{aligned}
& (\odot / \epsilon) \Theta^{*}\left(\Theta /(\mathbb{W})^{*}(\Theta / \Theta / \Theta)(\mathbb{W} /(\mathrm{U}))^{*}(\mathrm{E})^{*}(\odot / \epsilon)\right. \\
& (\odot / \epsilon) \Theta)^{*}(\oplus / \mathbb{T})(\mathbb{W} /(\mathrm{U}))^{*}(\mathrm{E})^{*}(\odot / \epsilon) \\
& \left.(\varangle / \epsilon)()^{*}(\mathbb{W} /(\mathbb{U}))^{*}(\mathbb{D}) \in\right)^{*}(\odot / \epsilon)
\end{aligned}
$$

Proof: Constraint 1 ensures that if there is a $\oslash$, then it must be the right-most particle in the chain. Similarly, if there is a $<$ then it is the left-most particle in the chain. The remaining states are all either lower case, upper case or control states, so well-formed states must be of the form

$$
(\lessdot / \epsilon)(\text { lowercase/Control/UPPERCASE })^{*}(\diamond / \epsilon) \text {. }
$$

Constrain 2 says that lower case sites must precede control sites which must preced upper case sites, so we have:

$$
(\varangle / \epsilon)(\text { lowercase })^{*}(\text { Control })^{*}(\text { UPPERCASE })^{*}(\diamond / \epsilon) \text {. }
$$

Constraint 3 enforces that there can be at most one consecutive control state which yields:

$$
(\lessdot / \epsilon)(\text { lowercase })^{*}(\text { Control } / \epsilon)(\text { UPPERCASE })^{*}(\diamond / \epsilon) \text {. }
$$

Constraint 4 says that if there are particles in a lower case or $<$ state and there are particles in a upper case or $\gg$ state, then there must be something to buffer them. This something can only be a control site because the configurations are restricted as indicated above. Thus we know the configuration must be a substring of:

$$
(\lessdot / \epsilon)(\text { lowercase }) *(\text { Control })(\text { UPPERCASE }) *(\diamond / \epsilon) \text {. }
$$

Constraints in item 5 ensure that within the lower case sites, (e) must precede (4) and $\mathbb{W}$ sites. Constraints in item 6 ensure that within the upper case sites, (U) and (W) sites must precede (E) sites. So a well-formed configuration must be a substring of:

$$
(\lessdot / \epsilon) \Theta)^{*}(\mathrm{U} / \mathrm{W})^{*}(\mathbf{C o n t r o l})(\mathrm{W} /(\mathrm{U}))^{*}(\mathrm{E})^{*}(\odot / \epsilon)
$$

If the control symbol is one of $\Theta, \Theta, \Theta$, there are no further constraints. If the control symbol is $(1)$ or $(\mathbb{I}$, then constraint 7 says that we have no (4) or (w) particles. If the control symbol is (1), then constraint 8 says that we have no (U) or (W) particles. 
Any state that corresponds to a configuration that is not well-formed will have an energy penalty from one of the terms from the first eight items in the list of illegal pairs. Thus, we can focus our attention on the well-formed states. The following observation follows from an inspection of the rules.

Observation 2.3 The set of well-formed states is closed under the transitions rules in both the forward and the backward directions.

Observation 2.3 and the following lemma show that the transition rules are well behaved on the set of well-formed states.

Lemma 2.4 For each well-formed state, at most one transition rule will apply in the forward direction and at most one will apply in the reverse direction.

Proof: We use the fact that a well-formed state has at most one site in a control state. Every transition rule, whether applied in the forward or reverse direction, involves a control site and a site to the immediate left or right. Furthermore, the type of control state uniquely determines whether it will be the site to the left or the right that it will be involved in the transition in the forward direction. The same is true for the reverse direction.

We now define a graph where each state in the standard basis is identified with a node in the graph and there is a directed edge from one state to another if there is a transition rule that takes one state to the other. We will call this graph the state graph for our construction. Observation 2.3 implies that the set of well-formed states is disconnected from the rest of the states. Furthermore, by Lemma 2.4, the graph when restricted to the set of well-formed states forms a set of disjoint directed paths. If there is a maximal path in the graph that has no illegal states, then a uniform superposition over those states is a zero energy eigenstate. Our next task is to characterize these paths. We would like to be able to say that the zero eigenstates are exactly those that correspond to the sequence of configurations illustrated earlier as our target ground state. Unfortunately, this is not necessarily true. For example, we could have a legal state which does not have a particle in a control state at all and this state will correspond to a single isolated node component of the graph. We can not enforce by local checks that a state has a control state. However, we will be able to make this assertion if we assume that the state begins and ends with $\diamond$ and $\gg$. Later we will need to add terms to our Hamiltonian to ensure the endpoints of the chain are in these delimiter states.

Definition 2.5 A standard basis state is bracketed if the leftmost particle is in state $<$ and the rightmost particle is in state $>$.

Note that the transition rules do not alter the number or locations of the $\gg$ and $\diamond$ sites, so the set of states in a path in the state graph are either all bracketed or all not bracketed. Thus, we can refer to a path as bracketed or not. Now we have several definitions that we will use to characterize the states in the target ground state. The first definition enumerates a set of properties that guarantee that the entangled pairs span the center of the chain.

Definition 2.6 A bracketed state is said to be balanced if it is well-formed and the following conditions hold:

1. Every site in state (W) or (W) occurs to the right of every site in state (U) or (4) in the chain.

2. If the control symbol is $\Theta$, $\Theta$ or (1) then the number or particles in state $(\ominus)$ is one more than the number of particles in (E) and the number of particles in state (U) or (u) is one less than the number of particles in state $\mathbb{W}$ or $\mathbb{W}$. 
3. If the control symbol is (II), (1) or $\Theta$, then the number or particles in state (e) is equal to the number of particles in state (E) and the number of particles in state (U) or (4) is equal to the number of particles in state $\mathrm{W}$ or $\mathrm{W}$.

4. If the control symbol is $\Theta$ there is one particle in state $(\Theta)$ and if the control symbol is $\Theta$, there are at least two particles in state (e).

5. If the control symbol is (II) there are no particles in state (e) and if the control symbol is (1) or $\ominus$, there is at least one particle in state (e).

The next definition will be used to ensure that the ground state is properly entangled. However, the property defined is not itself a quantum property in that it is defined for standard basis states which are represented by strings of symbols from the set of particle states specified in (1). The definition refers to the value of a qubit stored in a 2-state subsystem, but since we are referring to standard basis states, each qubit value is always 0 or 1 .

Definition 2.7 Consider a balanced state in the standard basis with $r$ particles in state $(\mathrm{E})$. The state is consistent if for $i=1$ to $r$, the $i^{\text {th }}$ site in state $(E)$ from the right end has the same qubit value as the $i^{\text {th }}$ state in (e) from the left. Furthermore, if the control symbol is $\Theta, \Theta$ or (1), then the qubit stored in the control state is the same as the qubit stored in the rightmost site in state $(e)$.

We will show that if a path in the state graph is composed of bracketed, balanced and consistent nodes then the first state in the path has the following configuration: $<$ (i) (U) ${ }^{m}(\mathbb{W})^{m}$. We say that any state that corresponds to this configuration is a good start state. The next lemma says that if a state is bracketed, balanced and consistent, then it belongs to a path whose initial state is a good start state. Furthermore the path is composed entirely of legal states. Then in the following two lemmas, we show that if a state is bracketed but not balanced or consistent, it belongs to a path that has at least one illegal state. This will leave three possibilities for a path: it is not bracketed, it it contains an illegal state, or it starts in a good start state and is composed entirely of legal states.

Lemma 2.8 Consider a bracketed, balanced and consistent state and the path $p$ in the state graph to which it belongs. The path p contains only legal states. Furthermore, the start state of $p$ is a good start state.

Proof: We will enumerate the possibilities for a bracketed, balanced and consistent state and show that after a transition rule is applied in either the forward or reverse direction, it remains bracketed, balanced and consistent. Furthermore, none of these states contains an illegal pair. We will also show that $<(\mathbb{1}) \mathbb{U}^{m} \mathbb{W}^{m}($ is the only bracketed, balanced, consistent configuration for which there is no tranisition rule that applies in the reverse direction. This makes it the only candidate for the first configuration in the path $p$. Let $m=(n-3) / 2$. We will refer to the sequence of (4), (U), (W) and $\mathbb{W}$ particles as the middle section We will break the argument down into cases, depending on the type of control symbol in the state:

(II): There is only one balanced configuration for this control state which is $<\left(\mathbb{1}(\mathrm{U})^{m}(\mathbb{W})^{m} \nabla\right.$. The only rule that applies to it does so in the forward direction and results in $<\odot \ominus \mathbb{U}^{m-1}(\mathbb{W})^{m} \gg$. This is a balanced configuration. Since the rule entangles the $(\ominus$ qubit with the $\Theta$, it is also consistent and legal.

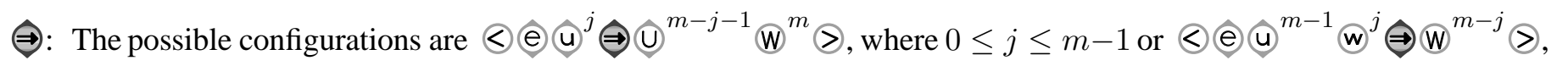
where $0 \leq j \leq m$. If the control state is at the left end of the middle section and the state is consistent, it will transition in the reverse direction to $\measuredangle(\mathbb{U}) \mathrm{U}^{m}(\mathrm{~W})^{m}($. If the control state is at the right end 
of the middle section, it will transition in the forward direction to $<$ (e) (4) $\left.)^{m}\right)^{m}(1)>$. Otherwise, when a transition rule is applied in the forward direction, the control state moves one site to the right and when a transition rule is applied in the reverse direction, it moves one site to the left. The state remains bracketed, balanced, legal and consistent.

(1): The configuration must have the following form: $<(\mathrm{e})^{i}(\mathrm{C}) \mathrm{UU}^{m-i}(\mathbb{W})^{m-i}(\mathrm{E})^{i} \otimes$, where $1 \leq i \leq m$. If $m=i$, there is no transition in the forward direction. If $m<i$, in the forward direction it goes to $<()^{i+1} \Theta(\mathrm{U})^{m-i-1}(\mathbb{W})^{m-i}(\mathrm{E})^{i} \gg$. The rule entangles the qubit values for the $\Theta$ and the rightmost $\left(\right.$ ), so the state remains consistent. In the reverse direction, it goes to $\odot()^{i} \Theta(\mathrm{U})^{m-i}(\mathrm{~W})^{m-i}(\mathrm{E})^{i} \curvearrowright$. The resulting states are bracketed, balanced, consistent and legal.

$\Theta$ : The first possible configurations is $\odot\left(\mathrm{e}^{i+1}(\mathrm{U})^{j} \Theta\left(\mathrm{U}^{m-j-i-1}(\mathbb{W})^{m-i}(\mathbb{E})^{i} \oslash\right.\right.$, where $1 \leq i \leq m-$ 1 and $0 \leq j \leq m-i-1$. The second is $\odot\left(\mathrm{e}^{i+1}(\mathrm{U})^{m-i-1} \mathrm{~W}^{j} \Theta \mathbb{W}\right)^{m-j-i}(\mathrm{E})^{i} \gg$, where $1 \leq$ $i \leq m-1$ and $0 \leq j \leq m-i$. If the control state is at theleft end of the middle section and the state is consistent, it will transition in the reverse direction to $<(e)^{i}(\mathbb{1}) \mathrm{U}^{m-i}(\mathbb{W})^{m-i}(\mathrm{E})^{i} \oslash$. If the control state is at the right end of the middle section, it will transition in the forward direction to $<(e)^{i+1}(4)^{m-i-1}(\mathbb{W})^{m-i}$ (1) $\left.E\right)^{i} \gg$. Otherwise, when a transition rule is applied in the forward direction, the control state moves one site to the right and when a transition rule is applied in the reverse direction, it moves one site to the left. The resulting states are bracketed, balanced, consistent and legal.

(1): The configuration looks like $\odot(e)^{i+1}$ (4) ${ }^{m-i-1}(\mathrm{~W})^{m-i}$ (1) $(\mathbb{E})^{i} \gg$, for $0 \leq i \leq m-1$. In the reverse direction, it transitions to $<()^{i+1}(\mathbb{U})^{m-i-1}()^{m-i} \Theta(\mathbb{E})^{i} \oslash$. In the forward direction, it transitions to $\left.\odot\left(e^{i+1}(4)^{m-i-1} \Theta\right)^{m-i-1} \ominus E\right)^{i+1} \oslash$. The forward transition rule transfers the qubit value from the (1) state to the leftmost (E), so it remains consistent. The resulting states are bracketed, balanced, consistent and legal.

$\Theta$ : The first possible configuration is $\odot\left(\mathrm{C}^{i}(\mathbb{U})^{m-i}(\mathbb{W})^{j} \Theta \mathbb{W}\right)^{m-j-i}(\mathbb{E})^{i} \gg$, where $1 \leq i \leq m$ and $0 \leq j \leq$ $m-i$. The second $\&()^{i}(\mathrm{u})^{j} \Theta(\mathrm{U})^{m-j-i}(\mathbb{W})^{m-i}(\mathrm{E})^{i} \gg$, where $1 \leq i \leq m$ and $0 \leq j \leq m-i$. If the control state is at the left end of the middle section and the state is consistent, it will transition in the forward direction to $<(e)^{i}(\mathrm{D}) \mathrm{UU}^{m-i}(\mathrm{~W})^{m-i}(\mathrm{E})^{i} \diamond$. If the control state is at the right end of the middle section, it will transition in the reverse direction to $\measuredangle\left(\mathrm{C}^{i}(\mathrm{U})^{m-i}(\mathrm{~W})^{m-i+1}\right.$ (D) $(\mathrm{E})^{i-1}(\nabla$. The (1) state takes the qubit value of the leftmost $(E)$ that it replaces and so the state remains consistent. Otherwise, when a transition rule is applied in the forward direction, the control state moves one site to the left and when a transition rule is applied in the reverse direction, it moves one site to the right. The state remains bracketed, balanced, legal and consistent.

Lemma 2.9 If a state in the standard basis is bracketed and well-formed but not balanced, it will evolve eventually (via forward or backwards rules) to a configuration which is not legal.

Proof: Starting with the first condition on balanced configurations, the only way for a configuration to have a (W) or (W) to the left of a (U) or (U) and not have an illegal pair from item 9 , is to have $(\mathbb{W}$ (Control) (U). Item 7 forbids (1)or (II)to the left of $(\mathrm{W}$ and item 8 forbids (1)to the left of a (U), so the control state must be one of $\Theta, \Theta$ or $\Theta$. In the next step, the configuration will transition to $\mathbb{W}(\mathbb{U})$ (Control) or (Control) (W) (U) which will create illegal pair (W) (U) or (W) (U) from item 9. 
Now let's assume that the condition on the $(\mathbb{E})$ and $(\odot)$ sites is violated. We will first address the problem that there are too many (e) sites. This will eventually evolve backwards to a configuration that looks like (<) (e)... (e) (4)... (4) $(\mathrm{w} \ldots$ (w) (1) $>$. Transitioning in the backwards direction, the $\gg$ site triggers the con-

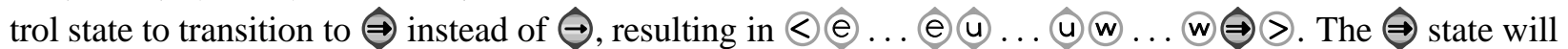

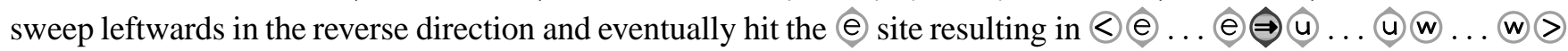

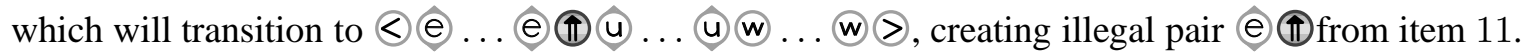

Similarly, if there are too many $(E)$ sites, we will eventually transition backwards to a configuration that

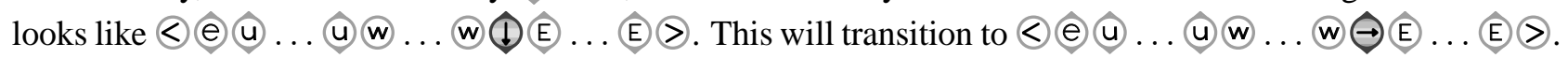

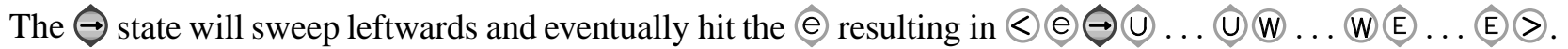
This transitions backwards to $<$ (1) (U) . . . (U) (W) ... (W) E . . E (E) $>$ which again creates illegal pair $<$ (1) from

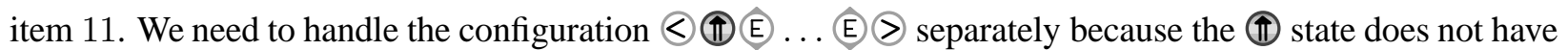
a transition in the reverse direction. However, this configuration is disallowed because the (II) $E$ pair is one of the illegal states pairs in item 11.

Now we will assume that the number of (E) sites and (e) sites are properly balanced. If we have too many (W) sites, we will eventually reach by forward transitions a configuration that looks like ... (e) (1) (U) W(W) E ... (with potentially more $\mathbb{W}$ sites). This configuration will evolve as follows:

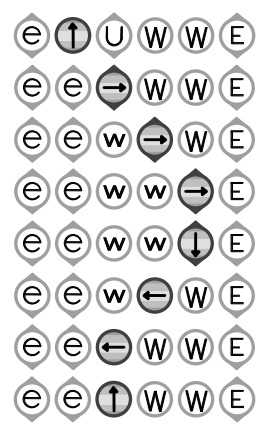

This creates illegal pair (1) Win item 12. Next we consider what happens if the number of (E) sites and (e) sites are properly balanced and we have too many (U) sites. We start with the case where there is a surplus of two or more (U) sites:

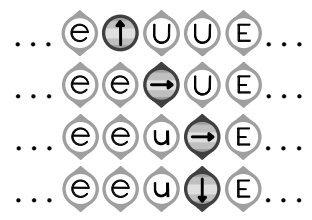

This creates illegal pair (U) (1)from item 12 . Now if there is only one extra (U) site:

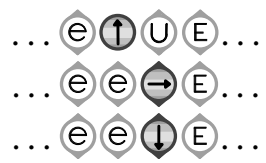

Once again, this creates illegal pair (e) (1) from item 12.

Finally, we handle the case where we have a double arrow instead of a single arrow (or vice versa). In the up-arrow case, $<(1)$ and (e) (11) are both illegal pairs from item 11, so the condition is checked locally. In the right-arrow case, if there is a configuration with a $\Theta$ and more than one $($ ), it will evolve by reverse transitions to $(e) \ominus$... which transitions to $(\ominus$ (II) (U)... which contains illegal pair $(e)$ (II) from item 11. 
Similarly, a state with one $(\ominus$ and a control in state $\Theta$ will transition in reverse to $\odot \odot \ominus$... which will go to $\measuredangle(1)$ (U) ... which contains illegal pair $\ll$ (1) from item 11 .

Lemma 2.10 If a state in the standard basis is bracketed and legal but not consistent, it will evolve eventually (via forward or backwards rules) to a configuration which is not legal.

Proof: Since the state is bracketed, we know that if it is not balanced, then it will evolve to an illegal state, so we can assume that the state is balanced but not consistent. This means that there must be a pair of (E) and (e) particles that don't have the same qubit but should. Eventually, we will transition backwards to this pair:

$$
\begin{aligned}
& \ldots \Theta_{0}(4) \ldots(\omega) \ldots \Theta \Theta(E) \Theta_{1}(E) \ldots \\
& \ldots \Theta_{0}(U) \ldots \text { (U) }\left(. . . \omega\left(\mathbb{W}_{1}(\mathrm{E}) \ldots\right.\right.
\end{aligned}
$$

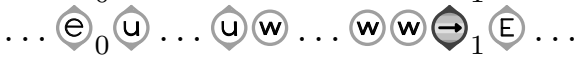

$$
\begin{aligned}
& \ldots\left(e_{0} \Theta_{1}(\mathrm{U}) \ldots \text { (U) W ... W W E } \ldots\right.
\end{aligned}
$$

This creates a violation with one of the constraints in item 13 . The result would be similar if the control states was $\Theta$ or the differing bits were swapped.

Now that we have characterized the paths in the state graph that are composed of legal configurations, we need to bound the spectral gap of $H_{\text {trans }}+H_{\text {legal }}$. We first need to bound the length of the paths.

Lemma 2.11 The length of any chain of well-formed states in the state graph is at most $n^{2}$, where $n$ is the number of particles in the chain.

Proof: We associate an ordered pair $(x, y)$ with every well-formed configuration, where $x$ is the number of sites in a ( $($ ) or a $(E)$ state. If the control site is in a state (1), (1) or (II), then $y=n$. If the control state is in state $\Theta$ or $\Theta$, then $y$ is the number of sites in state $(\omega)$ or $(\mathbb{W}$ that are to the left of the control state. If the control state is in state $\Theta$, then $y$ is the number of sites in state $(\mathrm{U})$ or $\mathbb{W}$ that are to the right of the control state. We define an ordering on these pairs by first comparing the first index. If the first index is the same, we compare the second index. It can be easily verified that if a transition rule applies to a configuration in the forward direction, the new configuration is associated with a pair of strictly greater value. Similarly, reverse transitions take a configuration to a configuration associated with a pair of strictly lesser value. Since there are at most $n^{2}$ possible pairs, the lemma follows.

Let $\mathcal{S}_{p}$ denote the space spanned by the basis states within a path $p$. Note that $\mathcal{S}_{p}$ is closed under $H_{\text {trans }}$ and $H_{\text {legal }}$. $H_{\text {legal }}$ when restricted to $\mathcal{S}_{p}$ and expressed in the standard basis is diagonal with non-negative integers along the diagonal. $H_{\text {trans }}$ when restricted to $\mathcal{S}_{p}$ and expressed in the standard basis has the form:

$$
\left(\begin{array}{rrrrrrr}
\frac{1}{2} & -\frac{1}{2} & 0 & & \cdots & & 0 \\
-\frac{1}{2} & 1 & -\frac{1}{2} & 0 & \ddots & & \vdots \\
0 & -\frac{1}{2} & 1 & -\frac{1}{2} & 0 & \ddots & \vdots \\
& \ddots & \ddots & \ddots & \ddots & \ddots & \\
\vdots & & 0 & -\frac{1}{2} & 1 & -\frac{1}{2} & 0 \\
& & & 0 & -\frac{1}{2} & 1 & -\frac{1}{2} \\
0 & & \cdots & & 0 & -\frac{1}{2} & \frac{1}{2}
\end{array}\right)
$$

We can now invoke Lemma 14.4 from [KSV02] to lower bound the energy of the overall Hamiltonian for a subspace $\mathcal{S}_{p}$ corresponding to a path with at least one illegal state. 
Lemma 2.12 Let $A_{1}, A_{2}$ be nonnegative operators, and $L_{1}, L_{2}$ their null subspaces, where $L_{1} \cap L_{2}=\{0\}$. Suppose further that no nonzero eigenvalue of $A_{1}$ or $A_{2}$ is smaller than $v$. Then

$$
A_{1}+A_{2} \geq v \cdot 2 \sin ^{2} \theta / 2
$$

where $\theta=\theta\left(L_{1}, L_{2}\right)$ is the angle between $L_{1}$ and $L_{2}$.

In our case, $A_{1}$ is the propagation Hamiltonian $H_{\text {trans }}$, and its null state, restricted to $\mathcal{S}_{p}$, is the equal superposition over all states in the path $p . A_{2}$ is the Hamiltonian $H_{\text {legal }}$, diagonal in the standard basis. Then $\sin ^{2} \theta$ is the fraction of illegal states in the path. The minimum nonzero eigenvalue of $H_{\text {legal }}$ is 1 , but (as in [KSV02]) the minimum nonzero eigenvalue of $H_{\text {trans }}$ is $\Omega\left(1 / K^{2}\right)$. In our case $K$, is the length of the path which by Lemma 2.11 is $O\left(n^{2}\right)$. Thus, if $p$ is a path containing an illegal state, all states in $\mathcal{S}_{p}$ have an energy at least $\Omega\left(1 / K^{3}\right)=\Omega\left(1 / n^{6}\right)$.

Before we summarize the results of this section, we will define a set of states which we will use to characterize the ground space of $H_{\text {trans }}+H_{\text {legal }}$. For each $x \in\{0,1,\}^{m}$, we define $\left|\phi_{x}\right\rangle$ to be the uniform superposition of the states in the path that begin with the state in configuration $<\left(\mathbb{N}(\mathbb{U})^{m}(\mathbb{W})^{m} \gg\right.$ whose qubit values in the (U) particles are set according to $x$.

Lemma 2.13 Consider the set of bracketed, well-formed states. Let $\mathcal{S}$ be the space spanned by these states. If $n$ is even, then the ground energy of $\left.\left(H_{\text {trans }}+H_{\text {legal }}\right)\right|_{\mathcal{S}}$ is $\Omega\left(1 / n^{6}\right)$. If $n$ is odd, the ground energy is 0 , the spectral gap is $\Omega\left(1 / n^{6}\right)$ and the null space is spanned be the $\left|\phi_{x}\right\rangle$.

Proof: Consider a path in the configuration graph consisting of well-formed, bracketed states. $H_{\text {trans }}+$ $H_{\text {legal }}$ is closed on the space spanned by the states in the path. If there is a state in the path which is balanced and consistent, then by definition $n$ must be odd. Furthermore, we know by Lemma 2.8 that the initial state in the path is a good start state and that the path contains no illegal states. The uniform superposition of all states in this path is an eigenstate of $H_{\text {trans }}+H_{\text {legal }}$ with zero energy.

If there is a state in the path which is either not balanced or not consistent, then by Lemmas 2.9 and 2.10, the path must contain an illegal state. Since the length of any path is at most $n^{2}$, the lowest eigenvalue in the subspace spanned by the states in this path is $\Omega\left(1 / n^{6}\right)$.

\subsection{Initializing Qubits}

We now add another term to each of the particles which will force the ground state to be a highly entangled state. This term is $\left|()_{-}\right\rangle\left\langle(\cup)_{-}\right|$. Expressing $\left|()_{-}\right\rangle$in terms of standard basis states, we get that $\left|()_{-}\right\rangle=$ $\frac{1}{\sqrt{2}}\left(\left|\cup_{0}\right\rangle-\left|\cup_{1}\right\rangle\right)$. Thus,

$$
\left|()_{-}\right\rangle\left\langle\left(\mathrm{U}_{-} \mid=\frac{1}{2}\left(| \mathrm { U } _ { 0 } \rangle \left\langle( \mathrm { U } _ { 0 } | - | \mathrm { U } _ { 0 } \rangle \left\langle( \mathrm { U } _ { 1 } | - | \mathrm { U } _ { 1 } \rangle \left\langle( \mathrm { U } _ { 0 } | + | \mathrm { U } _ { 1 } \rangle \left\langle\left(\mathrm{U}_{1} \mid\right) .\right.\right.\right.\right.\right.\right.\right.
$$

$H_{\text {init }}$ is the Hamiltonian obtained from summing this term as applied to all particles in the chain. Define

$$
\left|\phi_{g}\right\rangle=\frac{1}{2^{\frac{m}{2}}} \sum_{x \in\{0,1\}^{m}}\left|\phi_{x}\right\rangle .
$$

Lemma 2.14 Consider a quantum system consisting of a chain of n particles, where $n$ is odd. Let $\mathcal{S}$ be the space spanned by well-formed bracketed standard basis states. $H_{\text {trans }}+H_{\text {legal }}+H_{\text {init }}$ restricted to $\mathcal{S}$ has a spectral gap of $\Omega\left(1 / n^{6}\right)$ and $\left|\phi_{g}\right\rangle$ is its unique zero energy state. 
Proof: Since $H_{\text {init }}$ is non-negative, any state in $\mathcal{S}$ outside the space spanned by the $\left|\phi_{x}\right\rangle$ will have energy at least $\Omega\left(1 / n^{6}\right)$ by Lemma 2.13. The space spanned by the $\left|\phi_{x}\right\rangle$ is also spanned by a different basis: $\left|\phi_{a}\right\rangle$, where $a \in\{+,-\}^{m}$ and $\left|\phi_{a}\right\rangle$ is the uniform superposition of all states in the path whose starting state is the state in configuration $<\left(\mathbb{U}\left(\mathbb{U}^{m}\right)^{m} \mathbb{W}^{m} \gg\right.$ with the qubits in the (U) sites set according to $a$. The $\left|\phi_{a}\right\rangle$ are all zero eigenstates of $H_{\text {trans }}+H_{\text {legal }}$. Each $\left|\phi_{a}\right\rangle$ is also an eigenstate of $H_{\text {init }}$. The only $\left|\phi_{a}\right\rangle$ for which $H_{\text {init }}\left|\phi_{a}\right\rangle=0$ has $a=|+\rangle^{m}$ (which is exactly $\left|\phi_{g}\right\rangle$ ).

Now consider some $\left|\phi_{a}\right\rangle$ with $a \neq|+\rangle^{m}$. This state will violate $H_{\text {init }}$ in at least one term for at least the first state in the path. Since the path has length at most $n^{2}$, we know that $\left\langle\phi_{a}\left|H_{\text {init }}\right| \phi_{a}\right\rangle \geq 1 / n^{2}$. Thus, the energy penalty of $H_{\text {init }}$ for $\left|\phi_{a}\right\rangle$ is at least $1 / n^{2}$.

\subsection{Boundary Conditions}

We now want to add an energy term that will penalize states that are not bracketed. If we can use a positiondependent term on the first and the last particles in our chain, we could simply add the term $(I-|\measuredangle\rangle\langle\ll|-$ $|\gg\rangle\langle\rangle \mid)$ to the endpoints. This would add a penalty of at least one to any well-formed state which is not bracketed. The resulting Hamiltonian is frustration free.

Alternatively, we can apply the same term to every particle. $H_{\text {bracket }}$ is the Hamiltonian obtained from summing this term as applied to all particles in the chain. In order to do this, we need to weight $H_{\text {trans }}+$ $H_{\text {legal }}+H_{\text {init }}$ to ensure that we don't have endmarkers occurring in the middle of the chain.

Lemma 2.15 Let $H=3\left(H_{\text {trans }}+H_{\text {legal }}+H_{\text {init }}\right)+H_{\text {bracket, }}$, the unique ground state of $H$ is $\left|\phi_{g}\right\rangle$ and its spectral gap is $\Omega\left(1 / n^{7}\right)$.

Proof: Let $\mathcal{S}$ be the space spanned by the set of states in the standard basis that are well-formed and bracketed. $H$ is closed on $\mathcal{S}$. First we consider standard basis states outside of $\mathcal{S}$. If the state is not well-formed, it will have energy at least 3 from the $3 H_{\text {legal }}$ term. Let $s$ be the number of particles in a state $\diamond$ or $\diamond$ that are not one of the endpoints of the chain. Each one of these particles participates in at least one illegal pair and therefore contributes at least $3 / 2$ to the total energy from the $H_{\text {legal }}$ term. Therefore the state has a total cost of at least $3 \max \{s / 2,1\}$ from the $H_{\text {legal }}$ term. The energy from $H_{\text {bracket }}$ is at least $n-2-s$. Since $H_{\text {trans }}$ and $H_{\text {init }}$ are both non-negative, the energy is at least $n-2-s+3 \max \{s / 2,1\} \geq n-1$ for any standard basis state that is not well-formed. If a standard basis state is well-formed but not bracketed, it will have at most one $<$ or $>$ site. This comes from our characterization of well-formed states in Lemma 2.2 Thus, it will have energy of at least $n-1$ from the $H_{\text {bracket }}$ term.

Any state in $\mathcal{S}$ is an eigenstate of $H_{\text {bracket }}$ with eigenvalue $n-2$. The ground state of $H$ is still $\left|\phi_{g}\right\rangle$ but now with energy $n-2$ instead of 0 . Any other eigenstate in $\mathcal{S}$ has energy that is $\Omega\left(1 / n^{6}\right)$ from the $3\left(H_{\text {trans }}+H_{\text {legal }}+H_{\text {init }}\right)$ term which will give an overall energy of $(n-2)+\Omega\left(1 / n^{6}\right)$. Note that $\|H\|$ is $O(n)$. This comes from the observation that $H$ has energy $O(1)$ for each particle or pair of particles and there are $n-1$ neighboring pairs in the system. $H$ can then be normalized so that $\|H\|$ is $O(1)$ which will give a spectral gap of $\Omega\left(1 / n^{6}\right)$.

\subsection{Entropy of Entanglement}

We will use the following lemma several times in our discussion of the entanglement in the finite chain in this section and the discussion of the cycle in the next section.

Lemma 2.16 Let $\left|\psi_{i}\right\rangle$ for $1 \leq i \leq r$ be a set of states of a quantum system of $n$ particles. Let $A$ be $a$ subset of the particles and let $B$ be the complement of $A$. For each state $\left|\psi_{i}\right\rangle$, let $S_{i}$ be the set of standard basis states in the support of $\left|\psi_{i}\right\rangle$ and let $S_{i}^{A}$ be the resulting set when each state in $S_{i}$ is traced down to the particles in $A . S_{i}^{B}$ is the set resulting from tracing down the states in $S_{i}$ to the particles in $B . \rho_{i}$ is the 
density matrix for $\left|\psi_{i}\right\rangle$ and $\rho_{i}^{A}$ is the resulting state when $\rho_{i}$ is traced down to the particles in $A$. Define a new state $|\psi\rangle=\sum_{i=1}^{r} \alpha_{i}\left|\psi_{i}\right\rangle$. If all the $S_{i}^{A}$ are mutually disjoint or all the $S_{i}^{B}$ are mutually disjoint, then

$$
S\left(\rho^{A}\right) \geq \sum_{i=1}^{r}\left|\alpha_{i}\right|^{2} S\left(\rho_{i}^{A}\right) .
$$

Proof: Let's assume first that the $S_{i}^{B}$ sets are mutually disjoint. We will establish that $\rho^{A}=\sum_{i=1}^{r}\left|\alpha_{i}\right|^{2} \rho_{i}^{A}$. The lemma then follows from the fact that the entropy is concave.

$$
\rho=\sum_{i=1}^{r} \sum_{j=1}^{r} \alpha_{j}^{*} \alpha_{i}\left|\psi_{j}\right\rangle\left\langle\left.\psi_{i}\left|=\sum_{i=1}^{r}\right| \alpha_{i}\right|^{2} \rho_{i}+\sum_{i \neq j} \alpha_{j}^{*} \alpha_{i} \mid \psi_{j}\right\rangle\left\langle\psi_{i}\right| .
$$

The last sum consists of terms which are in turn sums over terms of the form $c|x\rangle\langle y|$, where $c$ is a complex number, $x \in S_{j}$ and $y \in S_{i}$ for $i \neq j$. We can express $x$ as $a_{x} b_{x}$ where $a_{x} \in S_{j}^{A}$ and $b_{x} \in S_{j}^{B}$. Similarly, we can express $y$ as $a_{y} b_{y}$ where $a_{y} \in S_{i}^{A}$ and $b_{y} \in S_{i}^{B}$. When we trace out the particles in $B$, the term $c|x\rangle\langle y|$ becomes $c\left|a_{x}\right\rangle\left\langle a_{y}\right|\left\langle b_{x} \mid b_{y}\right\rangle$. By assumption, $b_{x} \neq b_{y}$, so all of the terms in $\left|\psi_{j}\right\rangle\left\langle\psi_{i}\right|$ go to zero when $i \neq j$.

If we know that the $S_{i}^{A}$ sets are mutually disjoint, we can apply the result to the set $B$ and use the fact that $S\left(\rho_{j}^{A}\right)=S\left(\rho_{j}^{B}\right)$ for all $j$ and $S\left(\rho^{A}\right)=S\left(\rho^{B}\right)$.

Now we need to determine the entropy of entanglement for the ground state $\left|\phi_{g}\right\rangle$. We start by calculating the number of configurations in a path that begins with a good start state. We define an iteration to be the sequence of configurations beginning with the control particle in state (1) or (1) until it transitions to (1) again. The first configuration in the path has a (I) control state and the last has a (1) control state. If there are $m$ particles in state (U) at the beginning of an iteration, the iteration takes $4 m+1$ transitions. $m$ ranges from $(n-3) / 2$ down to 1 which gives $(n-3)^{2} / 2+3(n-3) / 2$ transitions and $T=(n-3)^{2} / 2+3(n-3) / 2+1$ configurations in the path.

We will need to divide the path into two parts since only the latter part of the path has high entanglement. We break the path at the point when the state has $(n-3) / 4+1$ particles in state $(e)$. Let $T_{1}$ denote the number of configurations in the first part of the path and $T_{2}$ the number of configurations in the second part of the path. The second part of the path corresponds to the last $(n-3) / 4$ iterations and so $T_{2}=$ $(n-3)^{2} / 8+3(n-3) / 4+1$. For every $n \geq 5$, there is some constant $c \geq 1 / 4$ such that $c T_{2}=T$. Let $\left|\phi_{1}\right\rangle$ denote a uniform superposition of the first $T_{1}$ configurations in the path and $\left|\phi_{2}\right\rangle$ the last $T_{2}$ configurations in the path. Recall that each configuration corresponds to a state which is a superposition of the $2^{m}$ basis states corresponding to the $2^{m}$ ways of setting the qubits in the 2 dimensional subsystems. Even if there are more than $m$ particles in states that can hold a qubit, we know that there are only $2^{m}$ ways to set the values of the qubits since we are guaranteed that the state is consistent (i.e. entangled pairs are really entangled). We have that

$$
\left|\phi_{g}\right\rangle=\sqrt{(1-c)}\left|\phi_{1}\right\rangle+\sqrt{c}\left|\phi_{2}\right\rangle
$$

where $\left\langle\phi_{1} \mid \phi_{2}\right\rangle=0$. All of the configurations in $\left|\phi_{2}\right\rangle$ start with $<(e)^{s+1} \ldots$, where $s=(n-3) / 4$. The configurations in the support of $\left|\phi_{1}\right\rangle$ have at most $s$ particles in state (e). This means that when we trace out at most $n-s-2$ particles on the right end of the chain, we can invoke Lemma 2.16. Thus, we can lower bound the entropy of entanglement for $\left|\phi_{2}\right\rangle$ which will serve to lower bound the entropy of entanglement for $\left|\phi_{g}\right\rangle$ to within a constant factor. Note that if $s<(n-3) / 4$ and we trace out $n-s-2$ particles, we need to break the path at the place where there are $s+1$ particles in state $(e)$, but the latter portion of the path will be larger and this will only serve to increase the value of $c$.

$\left|\phi_{2}\right\rangle$ is a uniform superposition of states in the standard basis. We can organize these into $2^{s}$ equally sized sets corresponding to the value of the qubits in the first $s$ particles in state $(e$. Since these first $s$ qubits are entangled with qubits on the right end of the chain, if we take two standard basis states from two 
different sets, these states must also differ somewhere in their last $s$ sites. Thus if we trace out $t$ particles on the right end of the chain for any $t \in\{s+2, n-s-2\}$, the resulting reduced density matrix expressed in the standard basis will be block diagonal with $2^{s}$ blocks each of which has a trace of $2^{-s}$. The entropy of the reduced density matrix is therefore at least $s$.

\section{Cycles and the Infinite Chain}

We now describe how to extend the construction for finite chains to cycles and the infinite chain. The parameter $n$ is no longer the number of particles in the system but just a parameter of the Hamiltonian that determines the spectral gap and a bound on the entanglement entropy in the ground state. We will assume throughout this section that $n$ is odd and that the number of particles in the cycle will be $n t$ for any $t \geq 2$. We will show bounds on the spectral gap and the entanglement entropy that are independent of $t$, so as $t$ goes towards infinity, the bounds will still hold which implies that they also hold for the infinite chain. The ground state is degenerate but any state in the ground space will exhibit entanglement entropy that is linear in $n$. As before, we describe a single two-particle term and apply that term to every neighboring pair on the cycle.

$H_{\text {trans }}$ remains unchanged, but we make several small changes to the Hamiltonian $H_{\text {legal }}$. The first change is that we allow the pair $\gg \diamond$. For a particular state, we will refer to a sequence of sites extending from a $\diamond$ site through the next $\gg$ site as a segment. The set of legal and well-formed states is exactly the same as it was for the finite chain except that we can now have more than one segment around the cycle. For example, we could have the following state wrapped around a cycle:

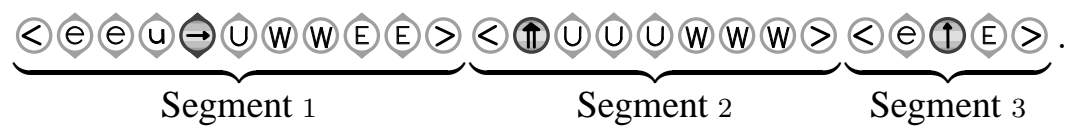

Note that it would be possible to replace the pair $\gg \odot$ by a single delimiting symbol, but it will be convenient to use the same notation we have developed in the previous section.

We will also add some additional illegal pairs. These are $\mathbb{T} \gg$ and anything of the form $\diamond X$ for any state $X$ not equal to (TiT) or (e). These additional illegal pairs serve to disallow segments of length two or three because a sequence of the form $\diamond X \gg$ or $\diamond \gg$ will contain an illegal pair. (The pair $(\ominus)$ is already disallowed in the original list of illegal pairs in item 4.) They have no effect on the ground state of $H_{\text {trans }}+H_{\text {legal }}$ for larger chains or segments since they never appear in the ground state configurations.

If a standard basis state is well-formed then every occurrence of $>$ has a $<$ to its immediate right and every occurrence of $\odot$ has a $\gg$ it its immediate left. Thus, we can assume that a standard basis state in the support of a ground state can be divided into valid segements. Of course, it is possible that there are no segments in which case the state could simply be a letter state (e.g., (e) or (W) repeated around the entire cycle. Later in this section we will introduce a term that will be energetically favorable to standard basis states that have at least one segment, so we will focus for now on those well-formed states that have at least one segment. Fix a set of locations for the $\gg \diamond$ pairs in the cycle, which will then determine the segments. Let $\mathcal{S}$ be the subspace spanned by all well-formed states in the standard basis that have these segments. $H_{\text {trans }}$ is closed over $\mathcal{S}$ as it was for the chain. The final Hamiltonian $H$ will be the sum of $H_{\text {trans }}$ and a set of terms which are all diagonal in the standard basis which means that $\mathcal{S}$ will also be closed under $H$ as well. We will characterize the eigenstates and corresponding eigenvalues of $H$ in $\mathcal{S}$.

Define $H_{\text {chain }}=H_{\text {trans }}+H_{\text {legal }}+H_{\text {init }}$. These are the terms that we borrow from the previous section on $1 \mathrm{D}$ chains (with the changes to $H_{\text {legal }}$ mentioned above). We will add in another Hamiltonian $H_{\text {size }}$ that will be designed to be energetically favorable to segments of size $n$. The final Hamiltonian $H$ will have the form $p(n) H_{\text {chain }}+H_{\text {size }}$ for some polynomial in $n$. 
Since all two-particle terms are zero on the pair $\diamond \diamond$, we can omit the two-particle terms which span two segments when considering $\left.H\right|_{\mathcal{S}}$. Now $H$ can be divided into a sum of terms, each of which acts on particles entirely within a segment. Let $H^{i}$ be the terms which act on particles within segment $i$. We can define $H_{\text {size }}^{i}$ and $H_{\text {chain }}^{i}$ similarly. An eigenstate of $H$ in $\mathcal{S}$ is then a tensor product of eigenstates of each $H^{i}$ acting on the particles in segement $i$. The energy is the sum of the energies of each $H^{i}$ on their corresponding eigenstate.

Consider $H_{\text {trans }}+H_{\text {legal }}+H_{\text {init }}$ from the previous section restricted to the subspace spanned by the set of all well-formed bracketed states acting on a chain of length $l$. This is exactly the same operator as $H_{\text {chain }}^{i}$ restricted to subspace $\mathcal{S}$, acting on the particles in a segment $i$ of length $l$ (with all other particles traced out). From Lemma 2.14, we know that if $l$ is odd, the spectral gap is $\Omega\left(1 / l^{6}\right)$ and there is a unique zero eigenstate $\left|\phi_{g}^{l}\right\rangle$ (with an additional parameter $l$ now denoting the length of the chain). If $l$ is even, then the minimum energy is $\Omega\left(1 / l^{6}\right)$.

We are now ready to define the final component of $H$. Recall that $T_{n}$ is the length of the path in the state graph corresponding to $\left|\phi_{g}^{n}\right\rangle$. In other words, $T_{n}$ is the number of configurations in the support of $\left|\phi_{g}^{n}\right\rangle$. In Section 2.3, we determined that $T_{n}=(n-3)^{2} / 2+3(n-3) / 2+1$.

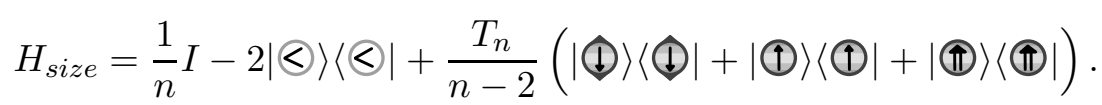

We will analyze the ground energy of a segment as a function of its length. We will need to use the Projection Lemma from [KKR06] which will allow us to focus on the ground space of $H_{\text {chain }}^{i}$.

Lemma 3.1 [KKR06] Let $H=H_{1}+H_{2}$ be the sum of two Hamiltonians acting on a Hilbert space $\mathcal{H}=\mathcal{T}+\mathcal{T}^{\perp}$. The Hamiltonian $\mathrm{H}_{2}$ is such that $\mathcal{T}$ is a zero eigenspace for $\mathrm{H}_{2}$ and the eigenvectors in $\mathcal{T}^{\perp}$ have value at least $J>2\left\|H_{1}\right\|$. Then

$$
\lambda\left(H_{1} \mid \mathcal{T}\right)-\frac{\left\|H_{1}\right\|^{2}}{J-2\left\|H_{1}\right\|} \leq \lambda(H) \leq \lambda\left(H_{1} \mid \mathcal{T}\right) .
$$

Corollary 3.2 There is a polynomial $p(n)$ such that $p(n)$ is $O\left(n^{10}\right)$ and for any segment of size $l \leq 2 n$ and $H^{i}=p(n) H_{\text {chain }}^{i}+H_{\text {size }}^{i}$

$$
\lambda\left(\left.H^{i}\right|_{\mathcal{S}}\right) \geq\left\langle\phi_{g}^{l}\left|H_{\text {size }}^{i}\right| \phi_{g}^{l}\right\rangle-1 / 2 n^{2} .
$$

Proof: We use the projection lemma with $H_{2}=p(n) H_{c h a i n}^{i}$ and $H_{1}=H_{\text {size }}^{i}$. Note that $H_{1}$ need not be positive, although it does need to be positive on $\mathcal{T}$ in order to yield a non-trivial lower bound. $\mathcal{T}$, the ground space for $H_{\text {chain }}^{i}$, is just the state $\left|\phi_{g}^{l}\right\rangle$. We need to establish that $\left\|H_{\text {size }}^{i}\right\|=O(n)$. Since $l \leq 2 n$, the first term is $O(n)$. The Hilbert space $\mathcal{S}$ is the set of all well-formed, bracketed states for that segement, so there can be at most one site in (1), (1) or (1) and at most one site in $<$. Thus the second two terms in $H_{\text {size }}^{i}$ are at most $1+T_{n} / n$ for any state in $\mathcal{S}$ which is also $O(n)$. The spectral gap of $H_{\text {chain }}^{i}$ is $\Omega\left(1 / n^{6}\right)$, so we can choose $p(n)$ so that $p(n)$ is $O\left(n^{10}\right)$ and $J$ (the spectral gap of $p(n) H_{\text {chain }}^{i}$ ) is at least $2 n^{2}\left\|H_{1}\right\|^{2}+2\left\|H_{1}\right\|$. Using Lemma 3.1, we can lower bound $\lambda\left(\left.H^{i}\right|_{\mathcal{S}}\right)$ by $\left\langle\phi_{g}\left|H_{\text {size }}^{i}\right| \phi_{g}\right\rangle-1 / 2 n^{2}$.

Note that we are not able to use the projection lemma for very large $l$ because the $\Omega\left(1 / l^{6}\right)$ gap will not be large enough. In the lemma below, we determine the ground energy of a segment as a function of its length. Large $l$ (greater than $2 n$ ) are dealt with separately with an argument that does not require the projection lemma.

Lemma 3.3 The operator $H^{i}$ acting on the l particles of segment $i$ restricted to well-formed bracketed states will have ground energy 0 and spectral gap $\Omega(1)$ for $l=n$. The ground energy is at least $1 / 2 n^{2}$ for any other value of $l$. 
Proof: Any sequence $\varangle X \gg$ will have an illegal pair. $\diamond \diamond$ is also illegal. Therefore, we can assume that $l \geq 4$. We consider four different cases based on the size of the segment $l$.

$\mathbf{l}=\mathbf{n}:$

Consider the state $\left|\phi_{g}^{n}\right\rangle .\left\langle\phi_{g}^{n}\left|H_{\text {chain }}\right| \phi_{g}^{n}\right\rangle=0$. Recall that $\left|\phi_{g}^{n}\right\rangle$ is a uniform superposition of states. There are $T_{n}$ distinct configurations represented in the support of $\left|\phi_{g}\right\rangle$ each of which as $2^{m}$ states for $m=(n-3) / 2$. Each configuration has one has a $\gg$ site and the number of configurations that contain a (1), (1) or (II) state is $n-2$. Therefore

$$
\left\langle\phi_{g}^{n}\left|H_{\text {size }}^{i}\right| \phi_{g}^{n}\right\rangle=\frac{n}{n}-2+\frac{T_{n}}{(n-2)} \frac{(n-2)}{T_{n}}=0 .
$$

Any state $|\psi\rangle$ that is orthogonal to $\left|\phi_{g}^{n}\right\rangle$ and is also in the subspace spanned by the well-formed states for segments of length $n$ will have $\left\langle\psi\left|p(n) H_{\text {chain }}\right| \psi\right\rangle \geq n^{4}$ and $\left\langle\psi\left|H_{\text {size }}\right| \psi\right\rangle \geq-2 n$. Thus, the spectral gap of $H^{i}$ will be $\Omega(1)$.

l > 2n:

Let $\psi$ be a state in the standard basis that is well-formed, bracketed and has length $l$. We will only lower bound $\left\langle\psi\left|H_{\text {size }}^{i}\right| \psi\right\rangle$. Since $H_{\text {chain }}^{i}$ is non-negative, the lower bound will hold for all of $H^{i}$. Furthermore, we will omit the last term in $H_{\text {size }}$ because this only adds to the energy. Every standard basis state in a bracketed well-formed segment of length $l$ has exactly one occurrence of $<$. Therefore the energy of a segment of length $l$ will be at least $l / n-2$. Since $l>2 n+1$, this will be at least $1 / n$.

$\mathbf{2 n} \geq \mathbf{l}>\mathbf{n}:$

We will first handle the case that $l$ is even. From Lemma 2.13, we know that the lowest eigenvalue of $H_{\text {trans }}+H_{\text {legal }}$ on a chain of length $l$ is $\Omega\left(1 / l^{6}\right)$ which is in turn $\Omega\left(1 / n^{6}\right)$. The other terms in $H^{i}$ are positive and the $H_{\text {trans }}+H_{\text {legal }}$ are weighted by a factor of $p(n)$ which bring the lowest energy to $\Omega(1)$.

Since we can assume that $l$ and $n$ are both odd, we know that $l \geq n+2$. We will use the projection lemma for this case and show that $\left\langle\phi_{g}^{l}\left|H_{\text {size }}^{i}\right| \phi_{g}^{l}\right\rangle \geq 1 / n^{2}$ which by Corollary 3.2 will be enough to lower bound $\lambda\left(H^{i}\right)$ by $1 / 2 n^{2}$. We osberve that

$$
T_{n}=\frac{(n-3)^{2}}{2}+\frac{3(n-3)}{2}+1=\frac{(n-1)(n-2)}{2}
$$

so $T_{n} /(n-2)=(n-1) / 2$, and

$$
\begin{aligned}
\left\langle\phi_{g}^{l}\left|H_{\text {size }}^{i}\right| \phi_{g}^{l}\right\rangle & =\frac{l}{n}-2+\left(\frac{T_{n}}{n-2}\right)\left(\frac{l-2}{T_{l}}\right) \\
& =\frac{l}{n}-2+\frac{n-1}{l-1} \\
& =\frac{l-n}{n}+\frac{n-l}{l-1} \\
& \geq(l-n)\left(\frac{1}{n}-\frac{1}{l-1}\right) \\
& \geq(l-n)\left(\frac{1}{n}-\frac{1}{n+1}\right) \geq \frac{2}{n(n+1)} \geq \frac{1}{n^{2}}
\end{aligned}
$$


$\mathbf{l}<\mathbf{n}:$

We can use the same reasoning as in the previous case to assume that $l$ is odd. Since both $l$ and $n$ are odd, we know that $l \leq n-2$. Now we will use the projection lemma for this case and show that $\left\langle\phi_{g}^{l}\left|H_{\text {size }}^{i}\right| \phi_{g}^{l}\right\rangle \geq 1 / n^{2}$ which will be enough to lower bound $\lambda(H)$ by $1 / 2 n^{2}$.

$$
\begin{aligned}
\left\langle\phi_{g}^{l}\left|H_{\text {size }}^{i}\right| \phi_{g}^{l}\right\rangle & =\frac{l-n}{n}+\frac{n-l}{l-1} \\
& \geq(n-l)\left(\frac{1}{l-1}-\frac{1}{n}\right) \\
& \geq(n-l)\left(\frac{1}{n-3}-\frac{1}{n}\right) \geq \frac{2}{n(n-3)} \geq \frac{1}{n^{2}}
\end{aligned}
$$

As a result of Lemma 3.3. we know that if the number of particles in the chain is $n t$, a multiple of $n$, and if $n$ is odd, then the ground energy of $H$ is zero and the ground space is spanned by the states that consist of $t$ copies of $\left|\phi_{g}^{n}\right\rangle$ tensored together. There are actually $n$ such states, which can be obtained by taking one and rotating it by one position $n$ times along the cycle. We will call these $\left|\psi_{0}\right\rangle, \ldots,\left|\psi_{n-1}\right\rangle$. Any eigenstate of $H$ that has a segment which is not equal to $n$ will have energy at least $\Omega\left(1 / n^{2}\right)$ while the norm of a single term in $H$ which acts on a pair of neighboring states is at most $O\left(n^{10}\right)$. This means the final spectral gap is $O\left(n^{-12}\right)$ when the Hamiltonian for the entire chain is scaled to $O(n)$. We still need to handle the case where there is a configuration which is well-formed but has no segments. This would just correspond to a configuration of all lower case states or all upper case states. $H_{\text {chain }}$ would evaluate to zero on such a state but $H_{\text {size }}$ would be at least $t$. Since these bounds are independent of $t$ and hold for arbitrarily large $t$, they hold as $t$ tends towards infinity.

\subsection{Entropy of Entanglement}

Consider the cycle with $n t$ particles, a basis state $\left|\psi_{i}\right\rangle$ and a set $A$ of contiguous particles in the cycle. We say that a particle in $A$ is good for $\left|\psi_{i}\right\rangle$ if it is the $p^{t h}$ particle in a some segment $j$ where $2 \leq p \leq n / 4$ or $3 n / 4 \leq p \leq n-1$ and the $(n-p)^{t h}$ particle in that segment is not in $A$.

Lemma 3.4 Consider a state $\left|\psi_{i}\right\rangle$ and a contiguous set $A$ of $r$ particles on the cycle. We assume that $r \leq n t-n$. When $\left|\psi_{i}\right\rangle$ is traced down to the particles in $A$, the entropy of the resulting state is at least the number of particles in $A$ that are good for $\left|\psi_{i}\right\rangle$ divided by 4.

Proof: The segments in $\left|\psi_{i}\right\rangle$ are fixed and we shall number them from 1 to $t .\left|\psi_{i}\right\rangle$ is a tensor product of states $\left|\psi_{i}^{j}\right\rangle$, where $\left|\psi_{i}^{j}\right\rangle$ is the ground state for a finite chain of length $n$ for segment $j$. The set of good particles can only come from two different segments. This is because if a segment contains a good particle, one of the endpoints in $A$ must be contained in that segment. We will arbitrarily call these segments $j$ and $k$. Let $A_{j}$ be the set of good particles in $j$ and $A_{k}$ be the set of good particles in $k$. The state $\left|\psi_{i}\right\rangle$ can be written as $\left|\psi_{i}\right\rangle=\left|\psi_{i}^{j}\right\rangle \otimes\left|\psi_{i}^{k}\right\rangle \otimes\left|\psi_{i}^{R}\right\rangle$. Where $\left|\psi_{i}^{R}\right\rangle$ is the state for the rest of the cycle (all sites not in segment $j$ or $k)$.

The support of $\left|\psi_{i}^{j}\right\rangle$ can be partioned into two sets depending on whether the good particles are all in an entangled state ( (e) or (E)) or whether there is a good particle that is not in an entangled state. Let $\left|\phi_{i}^{j}\right\rangle$ be the uniform superposition of the states in which all the good particles are entangled and $\left|\hat{\phi}_{i}^{j}\right\rangle$ be uniform superposition of the states for which there is a good site that is not in an entangled state.

Since the good particles are all either in the first $n / 4$ or last $n / 4$ particles in the chain, we can use the same argument used in Section 2.3 to determine that there is a constant $c_{j} \geq 1 / 4$ such that a fraction of $c_{j}$ 
of the states in the support of $\left|\psi_{i}\right\rangle$ are in the support of $\left|\phi_{i}^{j}\right\rangle$. We can write $\left|\psi_{i}^{j}\right\rangle=\sqrt{c_{j}}\left|\phi_{i}^{j}\right\rangle+\sqrt{1-c_{j}}\left|\hat{\phi}_{i}^{j}\right\rangle$ and $\left|\psi_{i}^{k}\right\rangle=\sqrt{c_{k}}\left|\phi_{i}^{k}\right\rangle+\sqrt{1-c_{k}}\left|\hat{\phi}_{i}^{k}\right\rangle . c_{k}$ is also at least $1 / 4$ although not necessarily equal to $c_{j}$.

$$
\left|\psi_{i}\right\rangle=\left(\sqrt{\left(1-c_{j}\right)\left(1-c_{k}\right)}\left|\hat{\phi}_{i}^{j}\right\rangle\left|\hat{\phi}_{i}^{k}\right\rangle+\sqrt{c_{j}\left(1-c_{k}\right)}\left|\phi_{i}^{j}\right\rangle\left|\hat{\phi}_{i}^{k}\right\rangle+\sqrt{\left(1-c_{j}\right) c_{k}}\left|\hat{\phi}_{i}^{j}\right\rangle\left|\phi_{i}^{k}\right\rangle+\sqrt{c_{j} c_{k}}\left|\phi_{i}^{j}\right\rangle\left|\phi_{i}^{k}\right\rangle\right) \otimes\left|\psi_{i}^{R}\right\rangle .
$$

Furthermore, the four states in the above sum satisfy the conditions for for Lemma 2.16 for the set $A$. $\left|\hat{\phi}_{i}^{j}\right\rangle\left|\phi_{i}^{k}\right\rangle\left|\psi_{i}^{R}\right\rangle$ has $\left|A_{k}\right|$ entangled pairs between $A$ and the rest of the cycle. Similarly, $\left|\phi_{i}^{j}\right\rangle\left|\hat{\phi}_{i}^{k}\right\rangle\left|\psi_{i}^{R}\right\rangle$ has $\left|A_{j}\right|$ entangled pairs and $\left|\phi_{i}^{j}\right\rangle\left|\phi_{i}^{k}\right\rangle\left|\psi_{i}^{R}\right\rangle$ has $\left|A_{j}\right|+\left|A_{k}\right|$ entangled pairs between $A$ and the rest of the cycle. We then have

$$
S\left(\rho_{i}^{A}\right) \geq c_{j}\left(1-c_{k}\right)\left(\left|A_{i}\right|\right)+\left(1-c_{j}\right) c_{k}\left(\left|A_{k}\right|\right)+c_{j} c_{k}\left(\left|A_{j}\right|+\left|A_{k}\right|\right) \geq \frac{1}{4}\left(\left|A_{j}\right|+\left|A_{k}\right|\right) .
$$

In the next lemma, we extend the lower bound on the entanglement to an arbitrary superposition of the $\left|\psi_{i}\right\rangle$.

Lemma 3.5 Consider a cycle with nt particles. Let $|\psi\rangle=\sum_{i=0}^{n-1} \alpha_{i}\left|\psi_{i}\right\rangle$. For any fixed $r \leq(n-1) t$, pick a random set $A$ of $r$ contiguous particles in the cycle. The expected entropy of entanglement of $|\psi\rangle$ when the state $|\psi\rangle$ is traced down to $A$ is at least $(\min \{r, n / 4\}-2) / 16$.

Proof: Consider a particular $\left|\psi_{i}\right\rangle$. With probability $1 / 4$, the left end of the segment will fall in the last $n / 4$ particles in a segment. If $r \leq n / 4$, this means that all but two of the particles are good (the exceptions are the sites in state $>$ and $<$ ). If $n / 4<r \leq n / 2$, then $n / 4-2$ of the particles are good. With probability $1 / 4$, the left end will fall in the range $n / 2+1$ to $3 n / 4$. If $r>n / 2$, then the number of good particles is at least $n / 4-1$ because $A$ will contain all of the particles in the last quarter of the segment. Since $r<n l-n$, it can not wrap around and contain any of the particles in the first quarter of that segment. Thus, with probability at least $1 / 4$, there are at least $\min \{r, n / 4\}-2$ good particles in $A$ for $\left|\psi_{i}\right\rangle$. Using Lemma 3.4, $E\left[S\left(\rho_{i}^{A}\right)\right] \geq(\min \{r, n / 4\}-2) / 16$.

Let $B$ be the complement of $A$ and $S_{i}^{B}$ be the set of standard basis states in $\left|\psi_{i}\right\rangle$ traced down to the set $B$. If $A$ has at most $n t-n$ particles then every state in every $S_{i}^{B}$ contains a $<$ site. Furthermore, for the states within a single $S_{i}^{B}$, the $<$ sites are the same and they are all different from the $<$ sites for the states in a $S_{j}^{B}$ for $i \neq j$. Thus, the $S_{i}^{B}$ 's are all mutually disjoint and we can apply Lemma 2.16 and linearity of expectations to get

$$
E\left[S\left(\rho^{A}\right)\right] \geq \sum_{i=1}^{r}\left|\alpha_{i}\right|^{2} E\left[S\left(\rho_{i}^{A}\right)\right] \geq \frac{\min \{r, n / 4\}-2}{16} .
$$

Since the random variable denoting the entropy of entanglement for a randomly chosen $A$ of size $r$ is in the range 0 to $\log (21 \cdot r)$, we can apply Markov's inequality to determine that with constant probability the entanglement entropy of a randomly chosen $A$ is $\Omega(\min \{r, n\})$.

Finally consider the translationally invariant state $|\Phi\rangle=\sum_{i=0}^{n-1}(1 / \sqrt{n})\left|\psi_{i}\right\rangle$. For any fixed set $A, A$ will have at least $\min \{r, n / 4\}$ good particles for at least $n / 4$ of the $\left|\psi_{i}\right\rangle$. Applying Lemmas 2.16 and 3.4 to these states, we get that the entanglement entropy of $A$ for $|\Phi\rangle$ is at least $(\min \{r, n / 4\}-2) / 16=\Omega(\min \{r, n\})$. 


\section{Open Questions}

There still remains an exponential difference in the dependence on $1 / \Delta$ between Hastings' area law and the lower bound presented here and that in [GH08]. Resolving this discrepancy may involve strengthening the upper bound given in the area law. There are also issues related to the translationally invariant construction given here that would be worthwhile to clarify. For example, is it possible to obtain a construction on the infinite chain that achieves the same entanglement entropy but with a unique ground state? Can one obtain a lower bound of $\Omega(\min \{m, n\})$ for the entanglement entropy on all regions of size $m$ instead of a constant fraction of the regions? Is there a 1D Hamiltonian for which the entanglement is linear in the region size simultaneously for all region sizes? The latter property could only be achieved on a gapless system because the 1D area law indicates that any non-zero spectral gap will imply a finite upper bound on the entanglement entropy for any region. It is not known whether this can be achieved even for a Hamiltonian with positiondependent terms. Finally, how robust are the entanglement properties in the ground state to small fluctuations in the terms of the Hamiltonian? It seems likely that the construction presented here will break with small errors in the transition rules. Is it possible to obtain a fault-tolerant version of this construction?

\section{Acknowledgements}

The author would like to thank Sergio Boixo, Daniel Gottesman, Matthew Hastings, Stephen Jordan and John Preskill for useful discussions.

\section{References}

[AGIK09] Dorit Aharonov, Daniel Gottesman, Sandy Irani, and Julia Kempe. The power of quantum systems on a line. Communications in Mathematical Physics, 287:41, 2009.

[AvDK ${ }^{+}$04] D. Aharonov, W. van Dam, J. Kempe, Z. Landau, S. Lloyd, and O. Regev. Adiabatic quantum computation is equivalent to standard quantum computation. In Proc. 45th FOCS, pages 4251. IEEE, 2004.

[ECP08] J. Eisert, M. Cramer, and M. B. Plenio. Area laws for the entanglement entropy - a review, 2008.

[FZ05] S. Farkas and Z. Zimborás. On the sharpness of the zero-entropy-density conjecture. Journal of Mathematical Physics, 46(12):123301-+, December 2005.

[GH08] Daniel Gottesman and Matthew Hastings. Entanglement vs. gap for one-dimensional spin systems, 2008. arXiv:0901.1108v1 [quant-ph].

[Has07] M. Hastings. An area law for one dimensional quantum systems. JSTAT, P08024, 2007. arXiv:0705.2024v2 [quant-ph].

[JWZ07] Dominik Janzing, Pawel Wocjan, and Shengyu Zhang. A single-shot measurement of the energy of product states in a translation invariant spin chain can replace any quantum computation, 2007. arXiv:0710.1615v2 [quant-ph].

[Kay08] Alastair Kay. The computational power of symmetric hamiltonians. Phys. Rev. A, 78:012346, 2008. arXiv:0801.3228v3 [quant-ph].

[KKR06] J. Kempe, A. Kitaev, and O. Regev. The complexity of the Local Hamiltonian problem. SIAM Journal of Computing, 35(5):1070-1097, 2006. 
[KSV02] A.Y. Kitaev, A.H. Shen, and M.N. Vyalyi. Classical and Quantum Computation. AMS, Providence, RI, 2002.

[LCV07] Y.-K. Liu, M. Christandl, and F. Verstraete. $N$-representability is QMA-complete. Phys. Rev. Lett., 98:110503, 2007. arXiv:quant-ph/0609125.

[Nag] Daniel Nagaj. Personal communication.

[NW08] Daniel Nagaj and Pawel Wocjan. Hamiltonian quantum cellular automata in 1d. Physical Review A, 78:032311, 2008.

[OT08] Roberto Oliveira and Barbara M. Terhal. The complexity of quantum spin systems on a twodimensional square lattice. COMP., 8:0900, 2008.

[Sch05] U. Schollwöck. The density-matrix renormalization group. Rev. Mod. Phys., 77:259-316, 2005 .

[Vid03] Guifre Vidal. Efficient classical simulation of slightly entangled quantum computations. Phys. Rev. Lett., 91:147902, 2003.

[Vid04] G. Vidal. Efficient simulation of one-dimensional quantum many-body systems. Phys. Rev. Lett., 93:040502, 2004.

[VLRK03] G. Vidal, J. I. Latorre, E. Rico, and A. Kitaev. Entanglement in quantum critical phenomena. Phys. Rev. Lett. ', 90, 2003.

[Whi92] S. R. White. Density matrix formulation for quantum renormalization groups. Phys. Rev. Lett., 69:2863, 1992.

[Whi93] S. R. White. Density-matrix algorithms for quantum renormalization groups. Phys. Rev. B, 48:10345, 1993. 